Z. klin. Chem. u. klin. Biochem.

8. Jg., S. $529-533$, September 1970

\title{
Die Kreatinineliminationsrate als Bezugsgröße für Analysen aus Harnproben
}

\author{
I. Einfluß der Harntagesmenge und des circadianen Rbytbmus auf die Kreatininausscheidung \\ Von D. Szadrowski, A. Jörgenseñ ${ }^{1}$ ), H.-G. Essing und K.-H. Schaller \\ Aus dem Institut für Arbeits- und Sozialmedizin der Universität Erlangen-Nürnberg \\ (Direktor: Prof. Dr. med. H. Valentin)
}

(Eingegangen am 26. Mai 1970)

\begin{abstract}
Um zu prüfen, ob sich die Kreatininausscheidung als Bezugsgröße bei Analysen aus Harnproben als genügend konstant erweist, wurde ihre Eliminationsrate bei 10 Probanden über mehrere Tage gemessen. An einem weiteren Kollektiv von 128 Personen wurde eine Beeinflussung der Kreatinintagesausscheidung durch Medikamente, die auf den Wasserhaushalt wirken, untersucht.

Es zeigte sich, daß die mittlere Kreatininausscheidung, ebenso wie die Harntagesmenge, über den Beobachtungszeitraum konstant war. Regressionsanalytisch ergab sich jedoch eine Abhängigkeit der Kreatininelimination vom Harnvolumen. Ähnliche Verhältnisse fanden sich unter Wirkung der verabfolgten Medikamente. Ein circadianer Rhythmus der Kreatininausscheidung ließ sich nicht objektivieren. Aufgrund dieser Ergebnisse sollte die Kreatininausscheidung nur dann als Bezugsgröße für Analysen aus Harnproben beibehalten werden, wenn der Quotient aus zu bestimmendem Parameter und Kreatininmenge eine genügende Konstanz aufweist.
\end{abstract}

\section{The rate of creatinine excretion as a reference value for the analysis of urine samples \\ I. The effect of daily urine volume and circadian rbythm on the excretion of creatinine}

In order to test whether the excretion of creatinine is sufficiently constant to act as a reference value for the analysis of urine samples, its elimination was measured over several days in 10 probands. The effect on the creatinine excretion of medicaments that act on the water balance was also tested on a group of 128 persons.

The average creatinine excretion and the daily urine volume were constant over the observation period. Regression analysis showed, however, that the elimination of creatinine depends on the urine volume. A similar relationship was found under the action of the medicaments. Creatinine excretion showed no circadian thythm.

On the basis of these observations, creatinine excretion can be used as a reference value for the analysis of urine samples, only if the quotient of the required parameter and the amount of creatinine can be shown to be sufficiently constant.

Bei arbeitsmedizinischen Überwachungsuntersuchungen ist man vielfach darauf angewiesen, die zu kontrollierende biochemische Größe im Harn zu bestimmen. In der Klinik bedient man sich üblicherweise für derartige Messungen der Tagesausscheidung im 24-Stdn.Harn. Am Arbeitsplatz jedoch ist eine 24stdg. Harnsammelperiode oft nicht durchführbar, so $d a ß$ sich als definierte Bezugsgröße für den interessierenden Parameter in Harnproben die Kreatininausscheidung eingebürgert hat.

Maßgebend für die Wahl des Kreatinins als Standardisierungsgröße war die von zahlreichen Autoren beschriebene Konstanz (Lit. bei 1 und 2) in der renalen Ausscheidung dieses Metaboliten. BoRTH und Mitarbeiter (3) halten jedoch aufgrund ihrer Untersuchungen die Kreatininausscheidung nicht für hinreichend gleichmäßig und messen insbesondere einer Variabilität der Kreatinineliminationsrate in Abhängigkeit vom Urinvolumen eine Bedeutung bei. Für bestimmte arbeitsmedizinische Fragestellungen, wie z. B. Untersuchungen an Hitzeexponierten, spielt jedoch gerade die wechselnde Flüssigkeitsaufnahme eine große Rolle. Gleichzeitig scheinen unter derartigen Arbeitsbedingungen endokrine Faktoren, die ebenfalls den Wasserhaushalt beeinflussen, wirksam zu werden (4).

1) D 29.
Unter diesen Aspekten sollte in der vorliegenden Arbeit einerseits geprüft werden, ob die Kreatininausscheidung für arbeitsmedizinische Belange weiterhin als Standardisierungsgröße angesehen werden kann. Zum anderen sollte insbesondere der Frage nachgegangen werden, inwieweit hormonell wirksame Medikamente, die gleichzeitig in den Wasserhaushalt eingreifen, die Kreatinineliminationsrate modifizieren können.

\section{Kreatin-Kreatininstoff wechsel und seine exogene Beeinflussung}

Kreatin wird im Aminosäurenstoffwechsel in Nieren und Leber gebildet, von wo es in die Muskelzellen gelangt. Dort dient es als Kreatinphosphat der Energiespeicherung. Der Kreatingehalt des Skelettmuskels beträgt etwa $20 \mathrm{mMol} / \mathrm{kg}$ (2). Durch Dehydratisierung wird irreversibel das innere Anhydrid des Kreatins, das Kreatinin, gebildet und über die Nieren ausgeschieden. Die Kreatininausscheidung ist nach MYERs (5) vom Gesamtkreatinin des Körpers und damit von seiner Muskelmasse abhängig. Es ist daher gebräuchlich, die Kreatininausscheidung auf das Körpergewicht zu beziehen (Kreatininkoeffizient, (6)). Auch die niedrigere Kreatininausscheidungsrate der Frauen $(1,2,7,8)$ wird durch eine geringere Muskelmasse erklärt. $\AA$ hnliches gilt für die Verminderung der Kreatininausscheidung mit zunehmendem Alter (8).

Die normale Kreatininausscheidung wird im allgemeinen für jedes Individuum als konstant angesehen. Sie beträgt $1-1,5 \mathrm{~g} / \mathrm{Tag}$ (2). Uber den Einfluß von Nahrungsaufnahme und Muskelarbeit auf die Kreatininausscheidung werden in der Literatur unter- 
schiedliche Ansichten vertreten. HOOGENHUYZE und Mitarbeiter (9), Myers (10) und Schulz (11) fanden einen Rückgang der Kreatininausscheidung bei Nahrungsentzug. Nach enteraler $\mathrm{Zu}-$ fuhr von Kreatinin durch Muskeleiweiß beobachteten KLERCKER (12) und VestergaArd (7) keine Zunahme der Kreatininausscheidung; ebenso bewirkt offenbar die orale Zufuhr reinen Kreatinins keine Anderung der Kreatininausscheidung (13). Dagegen fanden Hoogenhuyze (9), Myers (5), Zickedbein (14), BARRETT (15) und BLEILER (16) eine vermehrte Kreatininausscheidung nach gesteigerter Zufuhr von Kreatinin durch fleischhaltige Kost. Unter körperlicher Arbeit wiesen Schulz (11), Starling (17) und SRIVASTava (18) eine vermehrte Kreatininausscheidung nach, die dagegen von HoogenhuYze (9), HaLLAUer (19) und PATERson (20) auch unter körperlicher Schwerarbeit nicht objektiviert werden konnte.

Eine Beeinflussung der Kreatininausscheidung durch Schwankungen in der Flüssigkeitsbilanz ist im Gegensatz zu den oben angeführten exogenen Faktoren bisher nur vereinzelt untersucht worden (3).

\section{Methodik}

Stichprobenumfang und Untersuchungsgang

Zur Prüfung der in der Literatur beschriebenen Konstanz in der Kreatinintagesausscheidung wurde bei 10 gesunden berufstätigen Personen ( 6 Frauen, 4 Männer) im Alter von 27 bis 75 Jahren über 8 Tage die $24 \mathrm{stdg}$. Kreatininausscheidung im Harn bestimmt. Gleichzeitig wurde bei diesen Probanden untersucht, ob die renale Kreatininelimination einem circadianen $\mathrm{Rhythmus}$ unterliegt.

Weiterhin wurde bei 128 gesunden berufstätigen Personen ( 10 Frauen, 118 Männer) im Alter von 20-60 Jahren die Kreatininausscheidung im Harn vor und nach Applikation von Corticosteroiden bzw. deren Antagonisten und einem Hypophysenhormon bei sonst unveränderter Lebensweise bestimmt. Dieses Kollektiv wurde hinsichtlich der verabreichten Medikamente in fünf Gruppen aufgeteilt (Tab. 1).

Tab. 1

Aufteilung des Gesamtkollektivs hinsichtlich der applizierten Medikamente

\begin{tabular}{|c|c|c|c|c|}
\hline Gruppe & $\mathrm{n}$ & $0^{x} / 9$ & Medikament & $\begin{array}{l}\text { Dosis und } \\
\text { Applikation }\end{array}$ \\
\hline 1 & 30 & $\sigma^{\prime \prime}$ & $\begin{array}{l}\text { 6-Methyl-Prednisolon } \\
\text { (Urbason) }\end{array}$ & $\begin{array}{l}40 \mathrm{mg} \\
\text { i. } \mathrm{m} \text {. }\end{array}$ \\
\hline 2 & 25 & $\sigma^{\prime \prime}$ & $\begin{array}{l}\text { Metyrapon } \\
\text { (Metopiron) }\end{array}$ & $\begin{array}{l}6 \times 500 \mathrm{mg} \\
\text { per os }\end{array}$ \\
\hline 3 & 29 & $\sigma^{\prime}$ & $\begin{array}{l}\text { Aldosteron } \\
\text { (Aldocorten) }\end{array}$ & $\begin{array}{l}0,5 \mathrm{mg} \\
\text { i. } \mathrm{m} .\end{array}$ \\
\hline 4 & 24 & $\sigma$ & $\begin{array}{l}\text { Spironolactone } \\
\text { (Aldactone A) }\end{array}$ & $\begin{array}{l}4 \times 50 \mathrm{mg} \\
\text { per os }\end{array}$ \\
\hline 5 & $\begin{array}{l}10 \\
10\end{array}$ & $\stackrel{?}{\stackrel{9}{0}}$ & $\begin{array}{l}\text { adrenocorticotropes } \\
\text { Hormon } \\
\text { (Acortan prolongatum) }\end{array}$ & $\begin{array}{l}40 \text { I. E. } \\
\text { i. } \mathrm{m} \text {. }\end{array}$ \\
\hline
\end{tabular}

Die verwendeten Medikamente lassen sich wie folgt charakterisieren:

Acortin prolongatum, (adrenocorticotropes Hormon) stimuliert die Corticoidsynthese in der Nebennierenrinde. Das hauptsächlich gebildete Cortisol bewirkt u. a. eine vermehrte Natriumund Wasserretention.

Urbason, (6-Methyl-prednisolon; 11 $\beta, 17 \alpha, 21$-trihydroxy-6 $\alpha$ methyl-1,4-pregnadien-3,20-dion) induziert durch die Methylierung - im Gegensatz zu den genuinen Glucocorticosteroiden eine vermehrte Natriumausscheidung.

Metopiron, (2-Methyl-1,2-di-(4-pyridyl)-1-propanon) hemmt durch Blockierung der 11- $\beta$-Hydroxylase die Biosynthese von Cortisol, Aldosteron und Corticosteron. Der durch Metopiron hervor gerufene „Hypoaldosteronismus“ bewirkt u.a. eine intensive Diurese.

Aldocorten, (D-Aldosteron; 11 $\beta, 21-D i h y d r o x y-3,20$-dioxo-4 pregnen-18al) bedingt als das physiologische Mineralocorticoid eine vermehrte Natriumrückresorption im distalen Tubulus. Damit ist nịcht zwangsläufig eine vermehrte Wasserretention gekoppelt (22). Eine Wasserretention kommt erst sekundär über das antidiuretische Hormon zustande.

Aldactone $A$, (Spironolactone; 17-Hydroxy-7 $\alpha$-mercapto-3-oxo$17 \alpha$-pregn-4-en-21-carbonsäure, $\gamma$-lacton, acetat) bedingt durch kompetitive Hemmung der Aldosteron- bzw. Desoxycorticosteronwirkung am distalen Tubulus eine verminderte Natriumrückresorption. Gleichzeitig wird eine verstärkte Diurese eingeleitet.

Nach einer 24.stdg. Harnsammelperiode unter Basisbedingungen (Harn A) wurde das jeweilige Medikament appliziert und mit einer zweiten 24-Stdn.-Harnsammelperiode (Harn B) begonnen.

\section{Kreatininbestimmung}

Die Kreatininkonzentration wurde nach JAFFÉ (23) in Harn A und $\mathrm{B}$ bestimmt und die Kreatinintagesausscheidung unter $\mathrm{Be}$ rücksichtigung der $24 \mathrm{Stdn}$.-Harnmenge berechnet.

\section{Reagenzien}

Pikrinsäure (Merck 623) 1,2proz.

Natronlauge (Merck 6498) 10proz.

\section{Analysengang}

$1 \mathrm{~m} l$ Harn werden mit $20 \mathrm{~m} l$ Pikrinsäure-Lösung und $1,5 \mathrm{~m} l$ Natronlauge versetzt und nach 10 Min. mit dest. Wasser ad $100 \mathrm{~m} l$ aufgefüllt. Nach 5 Min. wird in $1 \mathrm{~cm}$ Schichtdicke bei $530 \mathrm{~nm}$ gegen einen Leerwert photometriert. Für den Leerwert wurde $1 \mathrm{~m} l$ dest. Wasser in gleicher Weise aufgearbeitet. Die Kreatininkonzentration ergibt sich in $\mathrm{mg} / 100 \mathrm{ml}$ durch Multiplizieren der Extinktion mit dem Faktor 245.

\section{Ergebnisse}

Die bei 10 Probanden über 8 Tage verfolgten Kreatininausscheidungen finden sich als Mittelwerte und Standardabweichungen für jeden der Untersuchten in Tabelle 2.

Tabelle 3 zeigt die für die einzelnen Tage gemittelten Werte der Kreatininausscheidung dieser 10 Probanden.

Tab. 2

Mittelwerte mit Standardabweichungen für Harnvolumen, Kreatininkonzentration und Kreatinintagesausscheidung bei 10 Probanden konzentration und Kreatinintagesaussch
über 8 Tage

\begin{tabular}{|c|c|c|c|c|c|c|c|}
\hline \multirow[t]{2}{*}{ Proband } & \multirow[t]{2}{*}{$\begin{array}{l}\text { Ver- } \\
\text { suchs- } \\
\text { zeitraum } \\
\text { [Tage] }\end{array}$} & \multicolumn{2}{|c|}{$\begin{array}{l}\text { Harnvolumen } \\
{[\mathrm{mll}]}\end{array}$} & \multicolumn{2}{|c|}{$\begin{array}{c}\text { Kreatinin- } \\
\text { konzentra- } \\
\text { tion } \\
{[\mathrm{g} / l]}\end{array}$} & \multicolumn{2}{|c|}{$\begin{array}{c}\text { Kreatinin- } \\
\text { tagesaus- } \\
\text { scheidung } \\
\text { [g/Tag] }\end{array}$} \\
\hline & & $\overline{\mathbf{x}}$ & $\pm s$ & $\overline{\mathbf{x}}$ & $\pm s$ & & $\pm s$ \\
\hline $\begin{array}{r}\text { II } \\
\text { III } \\
I V \\
\text { V } \\
\text { VI } \\
\text { VII } \\
\text { VIII } \\
\text { IX } \\
\mathbf{X}\end{array}$ & $\begin{array}{l}8 \\
7 \\
5 \\
8 \\
6 \\
8 \\
7 \\
8 \\
4 \\
5\end{array}$ & $\begin{array}{c}568,75 \\
882,86 \\
836,0 \\
619,38 \\
854,17 \\
1057,5 \\
717,14 \\
806,88 \\
1265,0 \\
1407,0\end{array}$ & $\begin{array}{r}129,86 \\
98,27 \\
51,28 \\
138,42 \\
239,76 \\
190,62 \\
74,99 \\
123,95 \\
220,08 \\
435,17\end{array}$ & $\begin{array}{l}1,79 \\
1,71 \\
1,49 \\
1,13 \\
1,32 \\
1,39 \\
1,59 \\
2,13 \\
0,97 \\
1,28\end{array}$ & $\begin{array}{l}0,43 \\
0,24 \\
0,22 \\
0,11 \\
0,3 \\
0,4 \\
0,12 \\
0,18 \\
0,28 \\
0,34\end{array}$ & $\begin{array}{l}0,97 \\
1,51 \\
1,25 \\
0,71 \\
1,07 \\
1,44 \\
1,14 \\
1,72 \\
1,19 \\
1,72\end{array}$ & $\begin{array}{l}0,21 \\
0,24 \\
0,21 \\
0,19 \\
0,11 \\
0,43 \\
0,1 \\
0,22 \\
0,19 \\
0,39\end{array}$ \\
\hline
\end{tabular}

Tab. 3

Mittelwerte und Standardabweichungen der Kreatininausscheidung über 8 Tage von 10 gesunden Erwachsenen

\begin{tabular}{|c|c|c|c|c|c|}
\hline & \multirow[b]{2}{*}{$\mathrm{n}$} & \multicolumn{2}{|c|}{$\begin{array}{l}\text { Kreatininkonzentration } \\
\qquad[\mathrm{g} / l]\end{array}$} & \multicolumn{2}{|c|}{$\begin{array}{c}\text { Kreatinintagesaus- } \\
\text { scheidung } \\
\text { [g/Tag] }\end{array}$} \\
\hline & & $\overline{\mathbf{x}}$ & $\pm s$ & $\overline{\mathbf{x}}^{\mathrm{l}}$ & $\pm s$ \\
\hline $\begin{array}{l}\text { 1. Tag } \\
\text { 2. Tag } \\
\text { 3. Tag } \\
\text { 4. Tag } \\
\text { 5. Tag } \\
\text { 6. Tag } \\
\text { 7. Tag } \\
\text { 8. Tag }\end{array}$ & $\begin{array}{r}10 \\
10 \\
10 \\
8 \\
6 \\
7 \\
8 \\
7\end{array}$ & $\begin{array}{l}1,59 \\
1,504 \\
1,314 \\
1,6175 \\
1,6217 \\
1,6057 \\
1,5238 \\
1,4529\end{array}$ & $\begin{array}{l}0,372 \\
0,3576 \\
0,3604 \\
0,4141 \\
0,2957 \\
0,4007 \\
0,3406 \\
0,7845\end{array}$ & $\begin{array}{l}1,286 \\
1,262 \\
1,2365 \\
1,1056 \\
1,1875 \\
1,34 \\
1,42 \\
1,2407\end{array}$ & $\begin{array}{l}0,2704 \\
0,469 \\
0,4358 \\
0,3763 \\
0,3788 \\
0,3765 \\
0,6126 \\
0,2733\end{array}$ \\
\hline
\end{tabular}


Tab. 4

Mittelwerte mit Standardabweichungen für Harnvolumen, Kreatininkonzentration und Kreatininausscheidung, A $=$ Vor Medikation, B = Nach Medikation. Vergleich der Mittelwerte im t-Test nach STUDENT

\begin{tabular}{|c|c|c|c|c|c|c|c|c|}
\hline \multirow{2}{*}{$\frac{\text { Gruppe }}{1}$} & \multirow{2}{*}{$\frac{\text { Medikament }}{\text { Urbason }}$} & \multirow{2}{*}{$\begin{array}{l}n \\
30 \\
30\end{array}$} & \multirow{2}{*}{$\begin{array}{l}\text { Harnvolumen [ml/Tag] } \\
\text { Kreatininkonzentration } \\
{[\mathrm{mg} / 100 \mathrm{ml}]}\end{array}$} & \multicolumn{2}{|c|}{ Vor $\frac{ \pm s^{M e c}}{295,25}$} & $\frac{\bar{x}}{1148,5}$ & $\begin{array}{r}\text { Nach } \\
s 92,01\end{array}$ & $\frac{t}{-1,7372}$ \\
\hline & & & & $\begin{array}{r}1000,0 \\
150,88 \\
1,436\end{array}$ & $\begin{array}{r}41,39 \\
0,368\end{array}$ & $\begin{array}{r}141,6 \\
1,563\end{array}$ & $\begin{array}{r}392,01 \\
40,03\end{array}$ & $\begin{array}{r}-1,7372 \\
1,1808 \\
-1,2832\end{array}$ \\
\hline 2 & Metopiron & $\begin{array}{l}25 \\
25 \\
25\end{array}$ & $\begin{array}{l}\text { Harnvolumen [ml/Tag] } \\
\text { Kreatininkonzentration } \\
\text { [mg/100 ml] } \\
\text { Kreatinintagesaussch. } \\
\text { [g/Tag] }\end{array}$ & $\begin{array}{l}1378,0 \\
131,26\end{array}$ & $\begin{array}{r}587,42 \\
46,043\end{array}$ & $\begin{array}{r}1827,2 \\
114,97\end{array}$ & $\begin{array}{r}873,92 \\
59,25\end{array}$ & $\begin{array}{c}-3,5084 * * \\
1,7896 \\
-0,9315\end{array}$ \\
\hline 3 & Aldocorten & $\begin{array}{l}29 \\
29 \\
29\end{array}$ & $\begin{array}{l}\text { Harnvolumen [ml/Tag] } \\
\text { Kreatininkonzentration } \\
\text { [mg/100 ml] } \\
\text { Kreatinintagesaussch. } \\
\text { [g/Tag] }\end{array}$ & 1049,83 & 265,18 & 1072,9 & $\begin{array}{l}37,79 \\
0,2657\end{array}$ & $\begin{array}{l}-0,4346 \\
-0,397 \\
-1,4382\end{array}$ \\
\hline 4 & Aldactone & $\begin{array}{l}24 \\
24\end{array}$ & $\begin{array}{l}\text { Harnvolumen }[\mathrm{m} l / \mathrm{Tag}] \\
\text { Kreatininkonzentration } \\
{[\mathrm{mg} / 100 \mathrm{ml}]}\end{array}$ & $\begin{array}{l}909,58 \\
135,56\end{array}$ & $\begin{array}{c}295,995 \\
52,27\end{array}$ & 1239,58 & 33,21 & $\begin{array}{c}-4,8434 * * * \\
2,7839 *\end{array}$ \\
\hline & & 24 & $\begin{array}{l}\text { Kreatinintagesaussch. } \\
\text { [g/Tag] }\end{array}$ & 1,1428 & 0,3379 & 1,2921 & $\dot{0,4004}$ & $-1,6927$ \\
\hline 5 & Acortan & $\begin{array}{l}20 \\
20 \\
20\end{array}$ & $\begin{array}{l}\text { Harnvolumen }[\mathrm{m} l / \mathrm{Tag}] \\
\text { Kreatininkonzentration } \\
\text { [mg/100 ml] } \\
\text { Kreatinintagesaussch. } \\
\text { [g/Tag] }\end{array}$ & $\begin{array}{r}166,15 \\
1,597\end{array}$ & $\begin{array}{l}52,54 \\
0,3763\end{array}$ & $\begin{array}{r}187,76 \\
1,6496\end{array}$ & $\begin{array}{l}71,23 \\
0,5113\end{array}$ & $\begin{array}{r}0,753 \\
-1,6394 \\
-0,8042\end{array}$ \\
\hline
\end{tabular}

$t *: p<0,05 ; t * *: p<0,01 ; t * * *: p<0,001$

Tab. 5

Mittelwerte mit Standardabweichungen des Harnvolumens, de Kreatininkonzentration und -tagesausscheidung unter Basisbedingungen für das Gesamtkollektiv

\begin{tabular}{rlcc}
\hline $\mathrm{n}$ & & $\overline{\mathrm{x}}$ & $\pm \mathrm{s}$ \\
\hline 138 & $\begin{array}{l}\text { Harnvolumen } \\
\text { [ml/Tag] }\end{array}$ & 1055,95 & 391,4429 \\
138 & $\begin{array}{l}\text { Kreatininkonzentra- } \\
\text { tion [g/l] }\end{array}$ & 1,4878 & 46,4619 \\
138 & $\begin{array}{l}\text { Kreatinintagesaus- } \\
\text { scheidung [g/Tag] }\end{array}$ & 1,464 & 0,38834 \\
\hline
\end{tabular}

Die bei den übrigen 128 Probanden vor und nach Medikation erhaltenen Werte der Harntagesausscheidung, deren Kreatininkonzentration und der Kreatinintagesausscheidung sind getrennt nach den beschriebenen Gruppen (Tab. 1) mit ihren Mittelwerten und Standardabweichungen sowie den entsprechenden $t$-Werten in Tabelle 4 wiedergegeben.

Um genauere Angaben über die normale mittlere Kreatininkonzentration bzw. Kreatinintagesausscheidung zu erhalten, wurden für diese Parameter Mittelwerte und Standardabweichungen aus den einzelnen Basiswerten des Gesamtkolletivs ( $n=138$ ) errechnet (Tab. 5).

\section{Diskussion}

Die in der vorliegenden Arbeit bei 138 gesunden Probanden gefundene mittlere Kreatinintagesausscheidung ( $T a b .5$ ) liegt mit 1,46 g/d innerhalb des in der Literatur angegebenen Normbereiches. Eine Beobachtung der Kreatininausscheidung von 10 Probanden über 8 Tage läßt erwartungsgemäß deutliche Unterschiede $z$ wischen den einzelnen Versuchspersonen erkennen (Tab. 2). Diese interindividuellen Diffe- renzen sind varianzanalytisch sowohl für die mittlere Kreatininkonzentration $\left.\left(\mathrm{F}=10,9613^{* *}\right)^{2}\right)$ als auch für die durchschnittlich eliminierte Kreatininmenge $(F=12,2706 * *)$ gesichert. Über den Beobachtungszeitraum dagegen zeigen Kreatininkonzentration und -menge in den Mittelwerten der 10 Probanden nur geringfügige Schwankungen (Tab. 3), die sich varianzanalytisch nicht sichern ließen $(F=0,5627$ bzw. $F=0,4040$ ). Damit scheint eine wichtige Voraussetzung zur Wahl der Kreatinineliminationsrate als Bezugsgröße für Analysen aus Harnproben, nämlich ihre Konstanz über verschiedene Tage, gegeben zu sein.

BORTH und Mitarbeiter (3) kommen allerdings aufgrund ihrer Untersuchungen zu der Auffassung, daß die Kreatinintagesausscheidung in Abhängigkeit vom Harnvolumen variiert. Daher wurde bei unseren Probanden ein Einfluß der Harntagesmenge auf die Kreatininausscheidung regressions- bzw. korrelationsanalytisch geprüft. Setzt man die Harntagesmengen während der untersuchten acht Tage bei den einzelnen Probanden in Beziehung $z u$ den ausgeschiedenen Kreatininmengen, so läßt sich trotz der kleinen Zahl (6 Freiheitsgrade) in zwei Fällen eine Abhängigkeit statistisch sichern $\left.\left(r=0,8357 * * \text { bzw. } r=0,7113^{*}\right)^{3}\right)$. Bei drei weiteren Probanden liegt der Korrelationskoeffizient nur geringfügig unterhalb der Grenze für eine Irrtumswahrscheinlichkeit von 5\%. Aufgrund der zugehörigen Regressionsfunktionen wird also bei der Hälfte der Probanden mit steigendem Harnvolumen

2) $\mathrm{F}^{* *}: \mathrm{p}<0,01$.

3) $\mathrm{r}^{* *}: \mathrm{p}<0,01 ; \mathrm{r}^{*}: \mathrm{p}<0,05$. 
eine zunehmende Menge Kreatinin ausgeschieden. Die oben beschriebene Konstanz der Kreatininelimination über den Beobachtungszeitraum ist dadurch zu erklären, daß das mittlere Harnvolumen (Tab. 2) ebenfalls keine Schwankungen aufweist $(F=0,8931)$. Insgesamt ist aber eine gleichbleibende Kreatinintagesausscheidung durch ihre Abhängigkeit vom Harnvolumen nicht immer gewährleistet. $\mathrm{Zu}$ ähnlichen Resultaten kamen MILLER und Mitarbeiter (24), BEST (25), FREMONT-SAITth und Mitarbeiter (26) sowie VesterGAARD und Mitarbeiter (7).

Auch die hier vorgelegten Ergebnisse der Untersuchungen über die Wirkung einiger den Wasserhaushalt beeinflussender Medikamente auf die Kreatininausscheidung bestärken die Vermutung einer Abhängigkeit der Kreatininausscheidung vom Harnvolumen. Das mittlere Harnvolumen stieg nach Gabe von Urbason, Metopiron und Aldactone an, wobei diese Zunahme für die Gruppen 2 und 4 statistisch gesichert ist (Tab. 4). Gleichzeitig ließ sich in allen Gruppen eine zwar geringe, aber deutliche Steigerung der ausgeschiedenen mittleren Kreatinintagesmenge beobachten. Setzt man die Harnmehrausscheidung unter der Medikation in Beziehung zu der Veränderung in der Kreatinintagesausscheidung der einzelnen Probanden, so errechnen sich die in Tabelle 6 wiedergegebenen Regressions-

Tab. 6

Regressionsfunktionen $\left(y^{\prime}=a+b x\right)$ und Korrelationskoeffizienten (r) für die Beziehungen zwischen der Zunahme der Harntagesmenge $(x)$ und der der Kreatinintagesmenge (y) unter Applikation der jeweiligen Medikamente

\begin{tabular}{cllrll}
\hline Gruppe & Medikament & $\mathrm{n}$ & $\mathrm{a}$ & $\mathrm{b}$ & $\mathrm{r}$ \\
\hline 1 & Urbason & 30 & $22,12+0,71$ & $0,6099 * * *$ \\
2 & Metopiron & 25 & $39,47+0,06$ & 0,1017 \\
3 & Aldocorten & 29 & $51,95+0,43$ & $0,5472 * *$ \\
4 & Aldactone & 24 & $-14,00+0,50$ & 0,3824 \\
5 & Acortan & 20 & $79,70+0,38$ & $0,51,44^{*}$ \\
\hline
\end{tabular}

r*: $\quad p<0,05$

r***: $:$ p $<0,001$

funktionen und Korrelationskoeffizienten. Letztere sind in den Gruppen 1, 3 und 5 statistisch signifikant von Null verschieden. Damit kann es als gesichert gelten, daß die durch diese Medikamente erzielte Mehrausscheidung an Harn mit einer gesteigerten täglichen Kreatininelimination einhergeht. Wenn man auch der Einwirkung von Urbason und ACTH einen gewissen katabolen Effekt zuschreiben muß, der evtl. die Kreatininmehrausscheidung bewirkt haben könnte, so gilt das nicht für Aldosteron, bei dem in gleicher Weise eine veränderte Kreatininausscheidung mit einem Unterschied in der Harnausscheidung korreliert.

In der Literatur werden $z$. T. circadiane Schwankungen in der Kreatininausscheidung beschrieben $(7,25,27,28$,
29 und 30). Diese Autoren gaben jedoch zum Teil nicht Kreatininkonzentrationen, sondern Kreatininmengen an. Darüber hinaus wurden statistische Sicherungsverfahren nicht angewendet.

Bei den hier durchgeführten Untersuchungen konnte ein Tagesrhythmus' der. Kreatininkonzentration im Harn an einem Kollektiv von 10 gesunden Erwachsenen nicht objektiviert werden. Wie Tabelle 7 zeigt, weisen

Tab. 7

Mittelwerte und Standardabweichungen der Kreatininkonzentration im Harn zu unterschiedlichen Tageszeiten bei 10 gesunden Erwachsenen

\begin{tabular}{ccccc}
\hline & $7^{00}-13^{00}$ & $13^{00}-19^{00}$ & $19^{00}-23^{00}$ & $23^{00}-7^{00}$ \\
\hline$\overline{\mathbf{X}}$ & 1,208 & 1,374 & 1,38 & 1,073 \\
{$[\mathrm{~g} / \mathrm{l}]$} & 0,369 & 0,4207 & 0,392 & 0,401 \\
$\begin{array}{c} \pm \mathrm{s} \\
{[\mathrm{g} / \mathrm{l}]}\end{array}$ & 0 & & \\
\hline
\end{tabular}

die mittleren Kreatinirikonzentrationen in vier Tagesabschnitten annähernd gleiche Werte auf. Der geringe Unterschied zwischen der Kreatininkonzentration der Nachtportion $(1,073 \mathrm{~g} / l)$ und den Nachmittags- bzw. Abendwerten (1,37 bzw. 1,38 g/l) läßt sich im t-Test nach STUDENT statistisch nicht sichern $(t=1,7314)$. Damit konnte das Ergebnis von Hale und Shannon (31) bestätigt werden, die ebenfalls keine signifikanten Unterschiede der Kreatininausscheidung zu verschiedenen Tageszeiten nachweisen konnten.

Das Fehlen eines Tagesthythmus in der Kreatininkonzentration des Harns wäre für das Heranziehen der Kreatinineliminationsrate als Bezugsgröße insofern vorteilhaft, als die Tageszeit für die Harnprobengewinnung vernachlässigt werden könnte. Dies hat insbesondere im Rahmen arbeitsmedizinischer Untersuchungen in Betrieben mit Schichtarbeit Bedeutung.

$\mathrm{Ob}$ andererseits die hier nachgewiesene Abhängigkeit der Kreatinineliminationsrate von der Harntagesmenge so gravierend ist, $\mathrm{da} ß$ die Kreatinintagesausscheidung als Bezugsgröße ungeeignet erscheinen muß, läßt sich durch die vorgelegten Ergebnisse allein nicht entscheiden. Eine Verfälschung der Ergebnisse wäre etwa dann möglich, wenn der Parameter, der auf Kreatinin bezogen werden soll, selbst einen ausgeprägten Tagesrhythmus aufweist oder mit dem Harnvolumen variiert. Die Kreatininausscheidung sollte demnach nur dann als Bezugsgröße für Analysen aus Harnproben beibehalten werden, wenn der Quotient aus zu bestimmendem Parameter und Kreatininmenge keine statistisch oder biologisch bedeutsame Abhängigkeit von der 24-Stdn.-Harnmenge zeigt. Zur Klärung dieser Frage sind entsprechende weitere Untersuchungen erforderlich.

\section{Literatur}

1. Flaschenträger, B. und E. Lehnartz, Physiologische Chemie. Der Stoffwechsel, II. Teil Springer Berlin (1957). - 2. RAPOPORT, S. M., Medizinische Biochemie, VEB Verlag Volk und Gesundheit Betlin (1964). - 3. BorTF, H., H. BreUER und D. GütGEMANN, diese Z. 6, 6 (1968). - 4. SzaDkowski, D., G. KohLER und G.
Lehnert, Ätztl. Forschg., Wörishofen 23, 271 (1969). - 5. MYers, V. C. und M. S. Fine, J. biol. Chemistry 14, 9 (1913). 6. Shafter, P. A., Amer. J. Physiol. 23, 1 (1908). - 7. VestergaArd,'P. und R. Leveretr, J. Laborat. Clin. Med., S. Louis, .51, 211 (1958). - 8. Ahrert, G., G. Brüschke, F. Dietze, H. 
Franke und J. HaAse, Zschr. Altersforsch. 20, 113 (1967). 9. HoogenhuYze, vaN, C. J. C. und H. Verplozgh, HoppeSeyler's Zschr. physiol. Chem. 46, 415 (1905). - 10. Mrars, V. C. und M. S. Fine, J. biol. Chemistry 15, 283 (1913). - 11. Schulz, W., Pflügers Arch. ges. Physiol: Menschen, Tiere 186, 126 (1921). 12. Klercker, K. O., Biochem. Z. 3, 45 (1907). - 13. HahN, A. und L. Schïrer, Zschr. Biol. 80, 195 (1924). - 14. ZrCkelaein, U., Zschr. exper. Med. 87, 112 (1933). - 15. BarretT, E. und T. AdDis, J. Clin. Invest. 26, 875 (1947). - 16. BleILer, R. E. und H. P. Schedr, J. Laborat. Clin. Med., S. Louis 59, 945 (1962). 17. Starding, E. K. und C. Lovatt Evans, Principles of human physiology. J. A. Churchill Ltd., London (1956). - 18. SRIvastava, S. S., K. V. Mani, C. M. Sont und J. BaAtr, Indian. J. Med. Res. 55, 953 (1967). - 19. Hallauer, W., H. Hauk und J. Schirmetster, Arch. Klin. Med. 213, 1 (1966/67). - 20. Paterson, N., Clin. Chim. Acta, Amsterdam 18, 57 (1967). 21. Herwig, B., Moderne Arzneimittel. Wissenschaftliche Ver- lagsgesellschaft, Stuttgatt (1967). - 22. KOCZOREK, K. H. R., M. v. BubNopf und G. RIECKER, in: KRücK, F. H., K. H. R. KoczoreK und G. Betzren: Klinische Anwendung der Aldosteron-Antagonisten. S. 111. Thieme, Stuttgart (1962). - 23. JAFṕ, M., Hoppe-Seyler's Z. physiol. Chem. 10, 399 (1886). 24. Miller, A. T., jr. und C. S. Blyth, J. Appl. Physiol. Washington 5, 73 (1952). - 25. Best, W. R., USA Med. Nutr. Lab. Rep. No. 118 (1953). - 26. Fremont-Smith, K., J. V. Carbone und I. C. Prough, Walter Reed Army Institute of Research Report Wrair 23 (1957). - 27. Addis, T., E. Barrett, L. J. Poo, H. J. UREEN und R. W. LIPPMAN, J. Clin. Invest. 30, 206 (1951). 28. Rosendaum, J. D., B. C. Ferguson, R. K. Davis und E. C. Rossmeisl, J. Clin. Invest. 31, 507 (1952). - 29. Plough, I. C. und C. F. Consolazio, J. Nutrit. 69, 365 (1959). - 30. Powell, R. C., I. C. Plough und E. M. BAker III: J. Nutrit. 73, 47 (1961). 31. Hale, J. B. und I. L. Shannon, Aerospace Med. 38, 1095 (1967).
Dr. D. Szadkowski 8520 Erlangen Schillerstr. 25 